\title{
Desempenho dos serviços de saúde em hospitais de Santa Catarina
}

A presente pesquisa buscou analisar o desempenho dos serviços de saúde prestados pelos 18 hospitais estaduais de Santa Catarina, nos quais a gestão é feita diretamente pelo Estado ou por Organizações Sociais de Saúde, utilizando o método estatístico denominado Análise Envoltória de Dados (DEA). Em Santa Catarina, são 5 hospitais geridos por OSS e se pretende estender este modelo organizacional para outros hospitais. Constatou-se que as OSS, em Santa Catarina, são, em média, $46 \%$ mais eficientes que os hospitais geridos pela administração direta. Os autores também recomendam uma política de investimentos que promova a adoção de arranjos organizacionais autônomos em todo hospital público novo. Embora os hospitais geridos por OSS tenham se mostrado superiores no que tange à eficiência do gasto público, observou-se que aspectos gerenciais também importam muito na qualidade do gasto, como foi evidenciado por alguns hospitais da administração direta com altos escores de eficiência.

Palavras-chave: Desempenho; Serviços de saúde; Hospitais

\section{Performance of health services in Santa Catarina hospitals}

This research sought to analyze the performance of health services provided by the 18 state hospitals in Santa Catarina, in which management is done directly by the State or by Social Health Organizations, using the statistical method called Data Envelopment Analysis (DEA). In Santa Catarina, there are 5 hospitals managed by OSS and it is intended to extend this organizational model to other hospitals. It was found that OSS, in Santa Catarina, are, on average, $46 \%$ more efficient than hospitals managed by direct administration. The authors also recommend an investment policy that promotes the adoption of autonomous organizational arrangements in every new public hospital. Although hospitals managed by OSS have been shown to be superior in terms of the efficiency of public spending, it was observed that managerial aspects also matter a lot in the quality of spending, as evidenced by some hospitals in the direct administration with high efficiency scores.

Keywords: Performance; Health services; Hospitals.

Topic: Políticas, Planejamento e Gestão de Sistemas e Serviços de Saúde

Reviewed anonymously in the process of blind peer.
Received: $\mathbf{2 1 / 0 2 / 2 0 2 0}$

Approved: 03/05/2020
Antonio Felipe Oliveira Rodrigues

Universidade do Estado de Santa Catarina, Brasil

http://lattes.cnpq.br/7704584890947372

http://orcid.org/0000-0001-9582-7438

felipenosul@gmail.com

Silvio Bhering Sallum (iD

Universidade do Estado de Santa Catarina, Brasil

http://orcid.org/0000-0003-4490-7598

sbs3y@virginia.edu

Fabiano Maury Raupp (iD)

Universidade do Estado de Santa Catarina, Brasil

http://lattes.cnpq.br/5713468691984238

http://orcid.org/0000-0001-9533-2574

fabianoraupp@hotmail.com
Referencing this:

RODRIGUES, A. F. O.; SALLUM, S. B.; RAUPP, F. M.. Desempenho dos serviços de saúde em hospitais de Santa Catarina. Scire Salutis, v.10, n.2, p.73-87, 2020. DOI: http://doi.org/10.6008/CBPC22369600.2020 .002 .0010 


\section{INTRODUÇÃO}

Grigoli et al. (2013), pesquisadores do Fundo Monetário Internacional, realizaram um estudo para mensurar o possível efeito nos indicadores da saúde ao se elevar tais despesas em países subdesenvolvidos. Os autores concluíram que o ganho de serviços de saúde para sociedade é superior em casos de choque de gestão quando comparados com aumentos das despesas públicas em saúde. Os resultados demonstraram que para os países de menor desenvolvimento relativo na amostra, ganhos de eficiência são capazes de elevar a expectativa de vida ajustada em até cinco anos, enquanto um aumento de dez por cento dos gastos públicos com saúde eleva a expectativa de vida em apenas dois meses.

Não há dúvidas de que a determinação da eficiência dos hospitais na produção, na alocação e no consumo de recursos aumentariam a produtividade no setor, e esta é uma etapa básica que deve ser considerada pelos formuladores de políticas. É necessário gastar melhor, ou seja, orientar os gastos segundo estudos de avaliação tecnológica e protocolos que indiquem a melhor forma de organizar e compor a gama de serviços na saúde, uma vez que o aumento dos recursos no setor por parte da administração pública é uma alternativa de mais difícil realização (GIOVANELLA et al., 2012).

Neste sentido, a presente pesquisa buscou analisar o desempenho dos serviços de saúde prestados pelos 18 hospitais estaduais de Santa Catarina, nos quais a gestão é feita diretamente pelo Estado ou por Organizações Sociais de Saúde, utilizando o método estatístico denominado Análise Envoltória de Dados (DEA). Com restrição orçamentária do setor público nos últimos anos, a área da saúde tem sido foco de reformas, dado o alto impacto e o grande volume de recursos que the é destinada, uma vez que a garantia deste direito como universal na Constituição Federal e a implementação do Sistema Único de Saúde (SUS) no Brasil reforçam a dimensão deste setor, sendo imperiosa para os gestores públicos a busca da introdução da cultura de eficiência e qualidade para uma crescente demanda a ser atendida.

\section{REVISÃO TEÓRICA}

É de suma importância entender o conceito de eficiência em si e as formas pelas quais é possível medi-la, principalmente considerando o contexto da saúde pública, porém é necessário ressaltar a diferença entre eficácia, produtividade eficiência. A primeira está ligada apenas ao que é produzido, sem levar em conta os recursos usados na produção (preocupa-se exclusivamente com o fim almejado). Já a produtividade é a razão entre o que foi produzido e o que foi gasto para produzir (se importa exclusivamente com o indivíduo).

Em relação a definição do termo eficiência, este analisa o que foi produzido, dado os recursos disponíveis, com o que poderia ter sido produzido com os mesmos recursos, isto é, a eficiência é uma medida relativa que tem relação com certas comparações de produtividade. Em momentos de crise econômica e uma grande escassez de recursos na saúde, medir a eficiência dos hospitais é a forma mais eficaz para estimular resultados ótimos com os atuais recursos disponíveis no setor. A avaliação de eficiência dos serviços de saúde se torna ainda mais relevante pelo fato de envolver grandes somas de recursos públicos para seu financiamento, uma vez que o setor de saúde é um dos principais receptores de recursos financeiros 
oriundos da administração pública brasileira, cerca de $13 \%$ apenas referente aos recursos estaduais de Santa Catarina (MARINHO et al., 2001).

Existem diversos métodos para medir a eficiência em um setor. Os métodos diferem, em geral, sobre as seguintes áreas: o tipo de medida que ele produz, o padrão de dados que ele demanda e aos pressupostos assumidos sobre a estrutura da tecnologia de produção e o comportamento econômico dos tomadores de decisão (COELLI et al., 2005). O setor hospitalar consiste em diversos insumos (em inglês, inputs) e geram diversos produtos (em inglês, outputs) e ainda possuem relações de economias e deseconomias de escala.

Dada tais especificidades do setor hospitalar, precisa-se da utilização de uma técnica estatística que considere as caraterísticas do setor. Inúmeros estudos nacionais e internacionais apontam a abordagem de modelagem denominada Análise Envoltória de Dados (DEA - Data Envelopment Analysis) como a mais apropriada para o presente fim. Segundo Kirigia et al. (2004), DEA é o método de investigação mais indicado para mensuração de eficiência na área da saúde, haja vista que possibilita a incorporação de múltiplos inputs e outputs e permite deferentes unidades de medidas. Complementarmente, Smith et al. (2005) argumentam que, frente à demanda pela avaliação e medição de eficiência dos serviços oriundos de recursos públicos, o método DEA é o mais indicado para suprir essa necessidade.

A Análise Envoltória de Dados é uma técnica não paramétrica, que, ao contrário das técnicas paramétricas, não especifica a priori os modelos de estrutura dos dados, permitindo que os modelos sejam determinados pelos próprios dados. Utiliza da programação linear matemática para classificar as unidades de acordo com uma pontuação de eficiência relativa. A natureza dos parâmetros é flexível e não fixada anteriormente à modelagem, como acontece em outras ferramentas estatísticas.

O DEA ordena as unidades produtivas por ordem de eficiência, e possibilita a medição dos insumos e produtos em unidades diferentes. Outra vantagem desta técnica é que ela não faz nenhuma suposição funcional prévia e considera que o máximo que poderia ter sido produzido é obtido por meio da observação das unidades produtivas, isto é, a pontuação máxima permitida pelo modelo é trucada na performance do melhor hospital analisado. Isto quer dizer que, em toda análise DEA, alguma unidade de análise sempre atingirá o ponto máximo de eficiência. Mas ressalva-se novamente que por este método a eficiência analisada é relativa. Pode haver várias outras técnicas e formas de gestão que façam os hospitais muito mais produtivos que o atual cenário, mas isto não é captado pelo modelo. Os valores resultados da análise DEA variam entre 0 e 1 , assim como um índice. Portanto, quanto mais próximo de zero, mais ineficiente é o hospital, e quanto mais próximo da unidade, mais eficiente ele é.

Além de emitir um escore de eficiência para cada hospital, o DEA também consegue dizer quais insumos são mal utilizados pelas unidades tomadores de decisão, e em que proporção está havendo este desperdício. Ou ainda, consegue medir quanto mais de produção o hospital poderia realizar, dados seus atuais insumos. Isto pode ser muito útil para estabelecer novas metas de gestão para os hospitais ineficientes, subsidiando estratégias de produção que maximizem a eficiência das unidades avaliadas e corrigindo ineficiências através de determinação de alvos. Por fim, por possuir dados dos hospitais de 2012 a junho de 2017, este relatório também pode atribuir importantes contribuições para a avaliação de 
diferentes técnicas de gestão ao calcular os índices de eficiência anuais deste período.

Entretanto, o DEA também possui limitações que são importantes ressaltar. Segundo La Forgia et al. (2008), os resultados são específicos para a amostra utilizada, não podendo ser utilizado para comparações entre unidades fora e dentro da amostra. O DEA não mede a eficiência absoluta dos hospitais, isto é, um hospital pode ocupar posições diferentes dependendo da amostra. Por fim, dados hospitalares estão sujeitos a problemas e erros de medição, assim como a ausência de informação abrangente e sistemática sobre custos por meio dos hospitais constitui um grande obstáculo para qualquer tipo de análise comparativa.

Recentemente, o DEA tem sido utilizado na área da saúde para estabelecer padrões de referência para hospitais (benchmarking), particularmente em países de desenvolvimento (GONÇALVES et al., 2007), seja para comparar índices de eficiência, seja para estabelecer benchmarking entre hospitais. No Brasil, entretanto, estudos empíricos sobre eficiência hospitalar ainda são raros. Até 2009, apenas nove estudos foram encontrados que utilizaram o DEA ou métodos similares de análises estatísticas para estimar a eficiência em hospitais (LA FORGIA et al., 2008). Cabe destacar que nos últimos anos a popularidade do DEA no Brasil tem aumentado consideravelmente, mesmo que seu uso ainda esteja isolado nas universidades. Um estudo de janeiro de 2018 elaborado por Emrouznejad et al. apresentou estatísticas do uso do DEA em publicações científicas pelo mundo desde 1978 até 2016. Em 1978 o modelo mais básico de DEA foi originalmente elaborado pelos matemáticos Abraham Charles, William Cooper e Edward Rhodes. A Figura 1 apresenta o resultado.

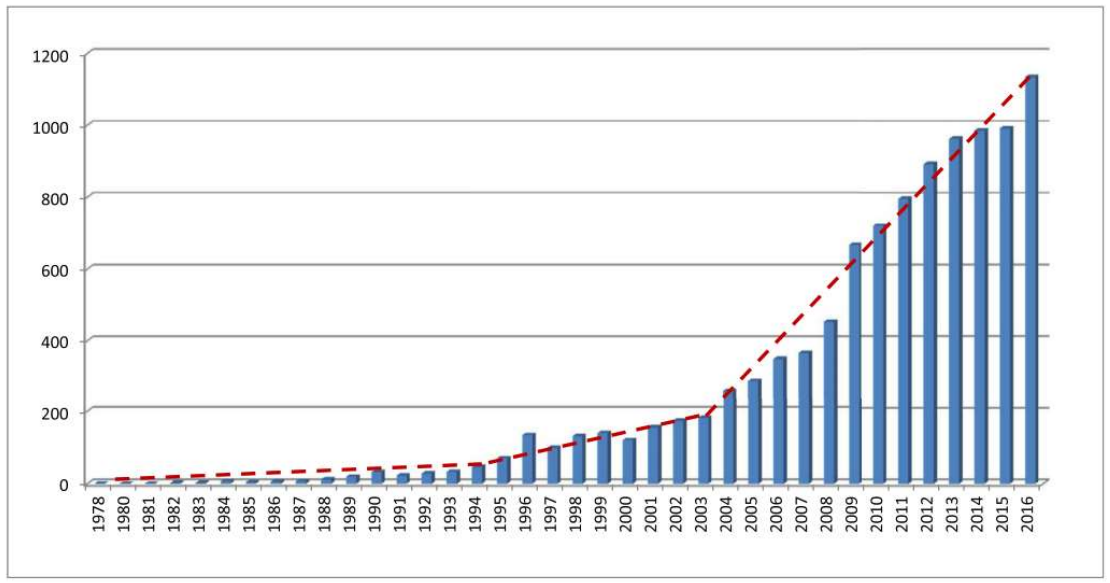

Figura 1: Utilização do DEA em publicações científicas de 1978 a 2016. Fonte: Emrouznejad et al. (2018).

Pela figura acima, nota-se que desde os anos 80 a análise de eficiência tem sido utilizada para avaliar o desempenho da produtividade de diversas áreas, porém na última década há um crescimento exponencial no uso do DEA, demonstrando a popularização do uso da técnica. Observa-se que o número de estudos nos últimos anos tem crescido drasticamente e importante periódicos como European Journal of Operational Research, Journal of the Operational Research Society e Journal of Productivity Analysis. São os que mais publicaram artigos que usaram DEA como ferramenta estatística. Eficiência, Tomada de Decisão, Eficiência Técnica e Programação Linear são os palavras-chave mais citadas nos artigos relacionados com o DEA. 


\section{METODOLOGIA}

Um termo muito utilizado na Análise Envoltória de Dados para representar as unidades tomadores de decisão (no caso do presente estudo, os hospitais), é 'DMU'. Quando temos vários tomadores de decisão como, por exemplo, empresas que produzem atividades parecidas, podemos comparar suas produtividades e descobrir o porquê de algumas serem mais produtivas que outras. De forma genérica, uma empresa é mais produtiva que outra porque tomou decisões que resultaram em maior produção, aproveitando então seus recursos de melhor forma. Essas decisões podem ser o uso de uma tecnologia mais avançada, a contratação de mão-de-obra mais qualificada, melhores técnicas gerenciais, entre outras razões. 0 importante é que a maior produtividade é, geralmente, decorrente de alguma tomada de decisão (MELLO et al., 2005). Daí o termo DMU, que corresponde a unidades tomadoras de decisão.

A Análise Envoltória de Dados utiliza inputs e outputs (entradas e saídas, respectivamente) para mensurar a eficiência comparativa que cada unidade individual mantém no seu processo de transformação (THANASSOULIS, 2001). Normalmente, os inputs são os fatores de produção como insumos, mão-de-obra e capital, e os outputs representam a produção das DMUs. Segundo La Forgia et al. (2008), o método DEA visa maximizar os produtos em relação a determinada quantidade de entradas, ou então minimizar a quantidade de recursos consumidos para um determinado nível de produto dependendo da orientação destinada à análise. Se o objetivo é maximizar os produtos mantendo constantes os recursos, efetua-se um DEA com orientação aos outputs. Mas quando o objetivo da análise é minimizar os recursos para atingir certo produto, efetua-se um DEA com orientação aos inputs.

A Figura 2 apresenta o gráfico onde o eixo $\mathrm{X}$ representa os insumos (inputs), o eixo $\mathrm{Y}$ representa a produção (outputs), e $f(x)$ a função da Fronteira de Possibilidade de Produção, que indica o máximo que pode ser produzido para cada nível de recurso, dado a tecnologia corrente. Embora esta figura represente um modelo básico bidimensional contendo somente um insumo e um produto, enquanto o DEA possibilita cálculos multidimensionais com vários insumos e produtos, este é um bom exemplo para entender melhor a dinâmica desta ferramenta estatística. Para iniciar a análise de eficiência, tome inicialmente o ponto $P$. Uma empresa que opere no ponto $P$ não é eficiente. Existem duas formas básicas de uma unidade não eficiente, tornar-se eficiente.

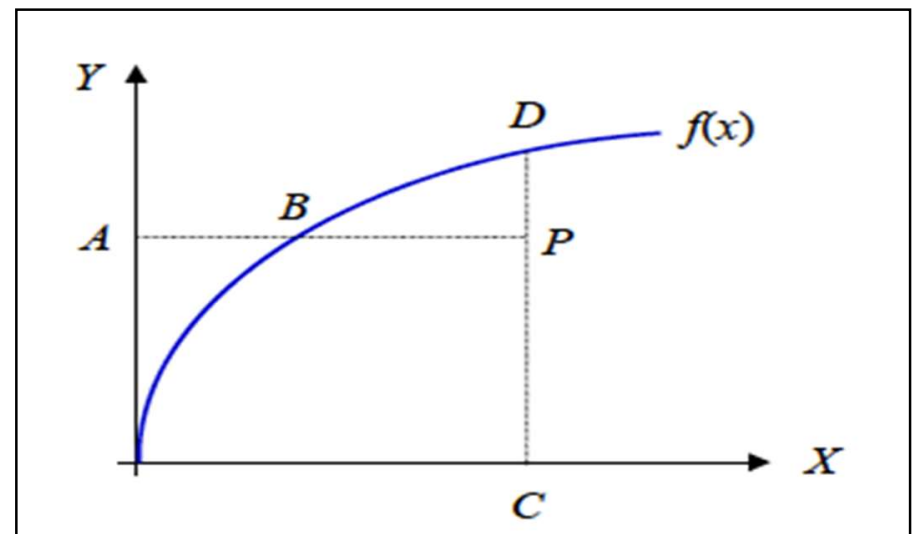

Figura 2: Fronteira de Possibilidade de Produção com o uso de dois insumos. Fonte: Mello et al. (2005). 
Se a DMU ineficiente que opera no ponto $P$ quiser maximizar sua eficiência pela orientação ao produto, ela precisa se deslocar até o ponto $D$, visto que a função de eficiência nos indica que uma empresa poderia produzir $D$ com os mesmos insumos que utilizados em $P$, que é a quantidade $C$ de insumos. A eficiência da empresa que opere em $P$, pela ótica do produto, será igual a razão CP/CD. Por outro lado, se ela quiser maximizar a sua eficiência pela orientação aos insumos, a empresa deve se deslocar até o ponto $B$, reduzindo seus insumos e mantendo seu produto constante em $A$. Por esta ótica, seu escore de eficiência seria o equivalente a razão $A B / A P$. Em ambos casos, a razão é um número entre 0 e 1 , sendo eficiente uma DMU que possua o escore igual a 1.

No caso de retornos variáveis de escala, que corresponde a uma variação não proporcional do produto a um aumento na quantidade de insumos, esta dupla orientação da análise de eficiência permite que rodemos a análise envoltória de dados duas vezes para cada ano, e que cada hospital tenha dois valores de eficiência diferentes para cada ano: um pela ótica do produto e outro pela ótica dos insumos. Entretanto, no caso em que os retornos de escala são constantes, os escores de eficiência por ambas orientações se coincidem (LA FORGIA et al., 2008). Não obstante, ressalva-se que mesmo com retornos constantes de escala, ainda é possível extrair conclusões acerca de quanto uma DMU necessita mudar para se tornar eficiente, seja reduzindo seus insumos, ou seja aumentando seu produto.

\section{RESULTADOS E DISCUSSÃO}

A Figura 3 apresenta a evolução, por hospital, da quantidade habilitada de leitos entre 2012 e 2017.

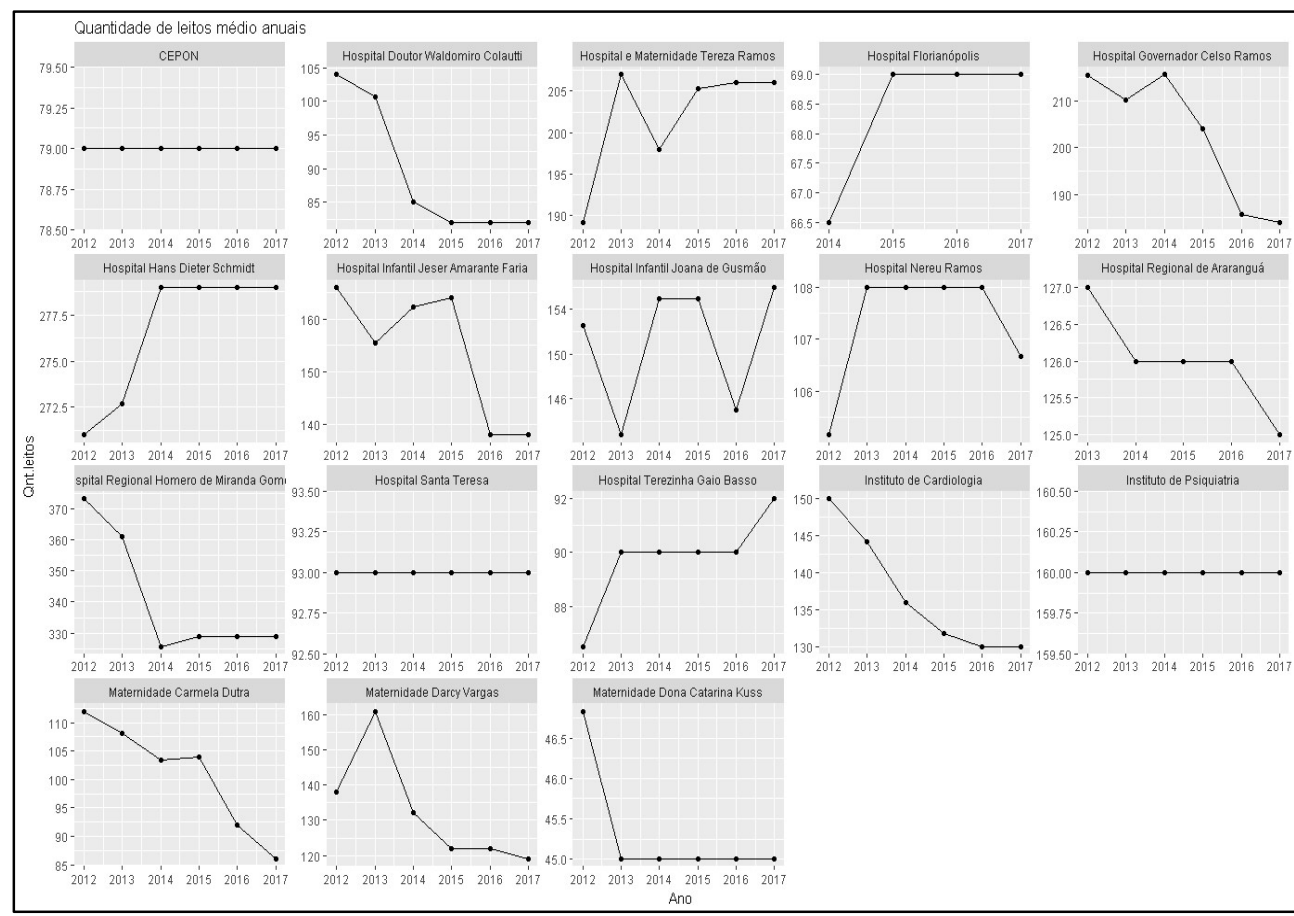

Figura 3: Quantidade de leitos habilitados por hospital, de 2012 a 2017. Fonte: Elaborada a partir de dados da Secretaria Estadual de Saúde de Santa Catarina (SESSC) e do Cadastro Nacional de Estabelecimento de Saúde (CNES).

Nota-se que CEPON, Hospital Santa Tereza e Instituto de Psiquiatria mantiveram a mesma quantidade de leitos habilitados durante o período. Já os hospitais Doutor Waldomiro Colautti, Celso Ramos, 
Hospital Infantil Jeser Amarante Faria, Hospital Regional Homero de Miranda Gomes, Instituto de Cardiologia, Maternidade Carmela Dutra e Maternidade Darcy Vargas apresentaram quedas no número de leitos. As unidades hospitalares Maternidade Tereza Ramos, o Hospital Florianópolis e o Hospital Terezinha Gaio Basso apresentaram o maior aumento. A evolução ao longo da relação de gastos por leito é apresentada na Figura 4.

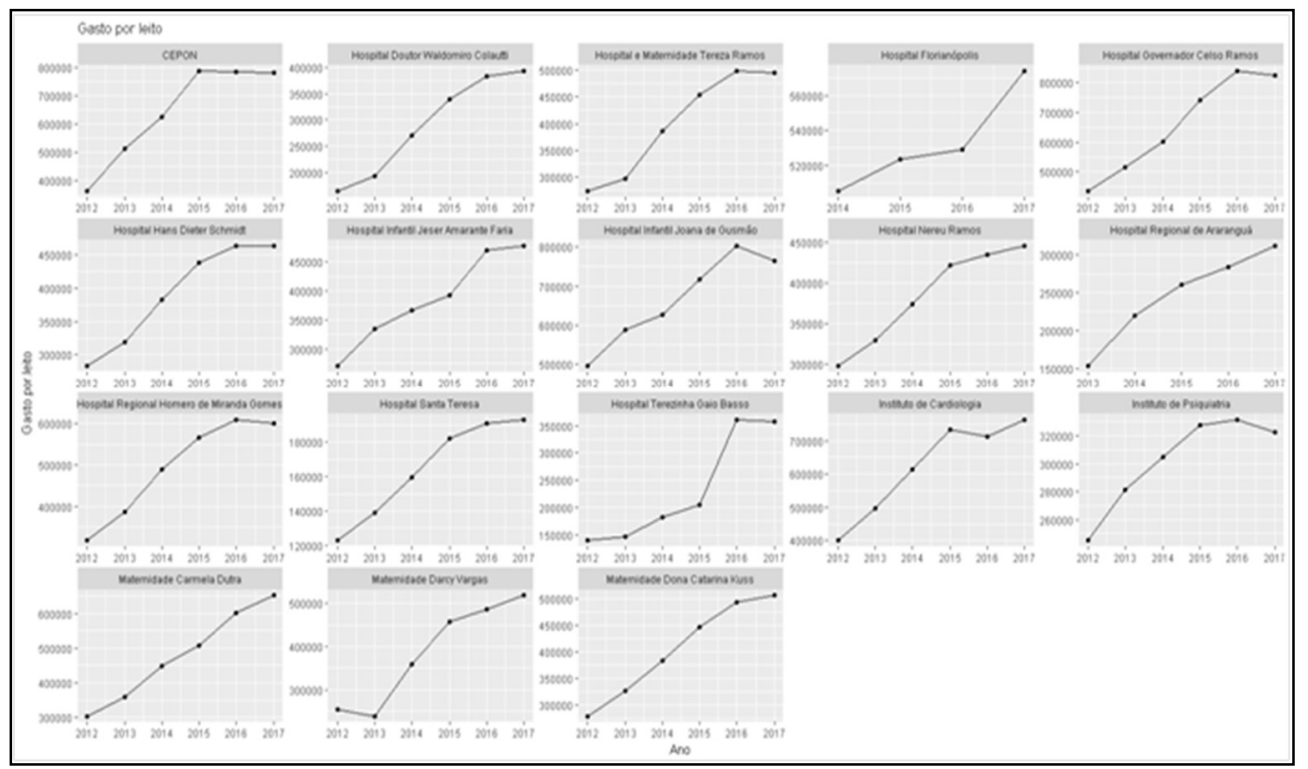

Figura 4: Evolução, por hospital, do gasto por leito entre 2012 e 2017. Fonte: Elaborada a partir de dados da SESSC e do CNES.

Analisando a figura acima, evidencia-se que o aumento expressivo no gasto por leito foi unânime em todos os hospitais durante o período observado. Analisando mais minuciosamente, conclui-se que estes aumentos foram todos superiores à inflação do IPCA acumulada entre 2012 e metade de 2017, que foi de aproximadamente 40\%. A Figura 5 apresenta a evolução, por hospital, do número de médicos entre 2012 e 2017.

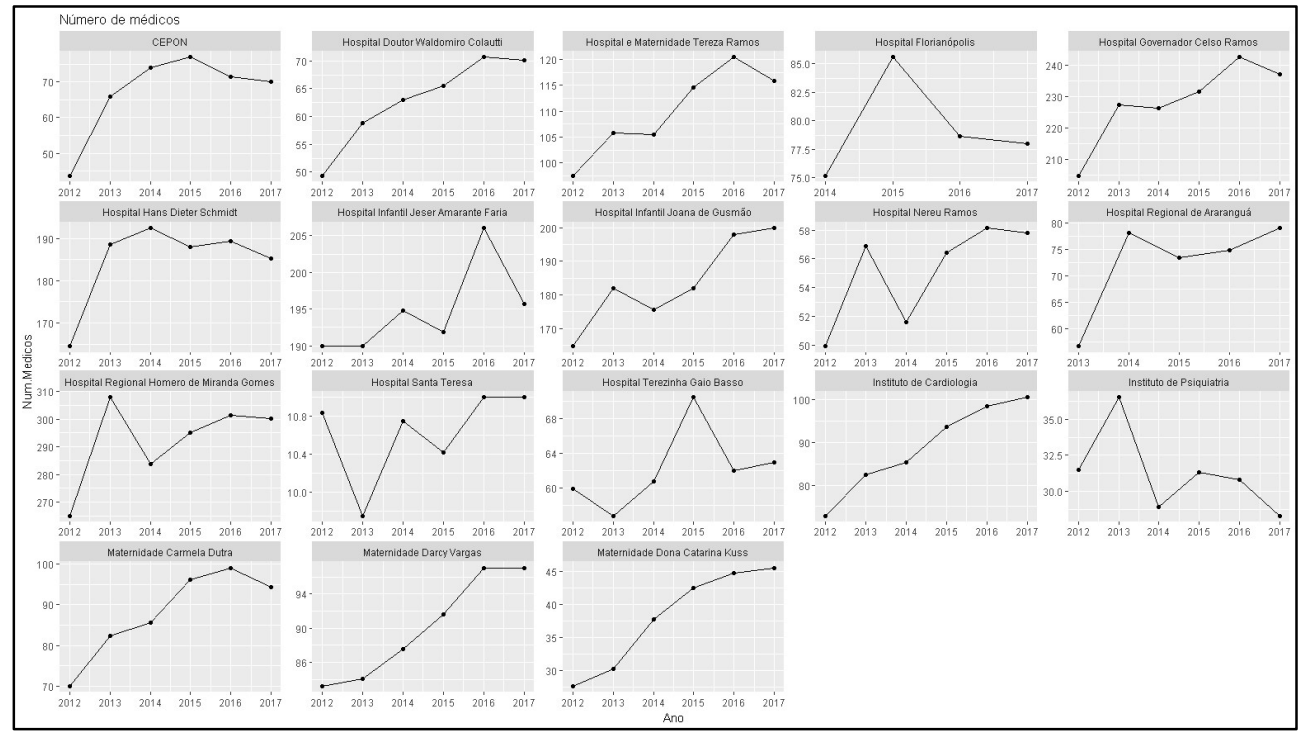

Figura 5: Evolução, por hospital, do número de médicos entre 2012 e 2017. Fonte: Elaborada a partir de dados da SESSC e do CNES.

Com exceção do Instituto de Psiquiatria, que em 2017 apresentou valor similar ao de 2012, todos outros hospitais aumentaram o número de médicos ativos no período analisado. CEPON, Hospital Doutor 
Waldomiro Colautti, Hospital e Maternidade Tereza Ramos, Hospital Celso Ramos, Hospital Hans Dieter Schmidt, Hospital Infantil Joana de Gusmão, Hospital Regional de Araranguá, Hospital Homero de Miranda Gomes, Instituto de Cardiologia, e as Maternidades Carmela Dutra, Darcy Vargas e Dona Catarina Kuss todos contrataram, no mínimo, 10\% a mais de médicos no final do período em relação à média de 2012 . A Figura 6 apresenta a evolução, por hospital, do número de outros funcionários da saúde entre 2012 e 2017.

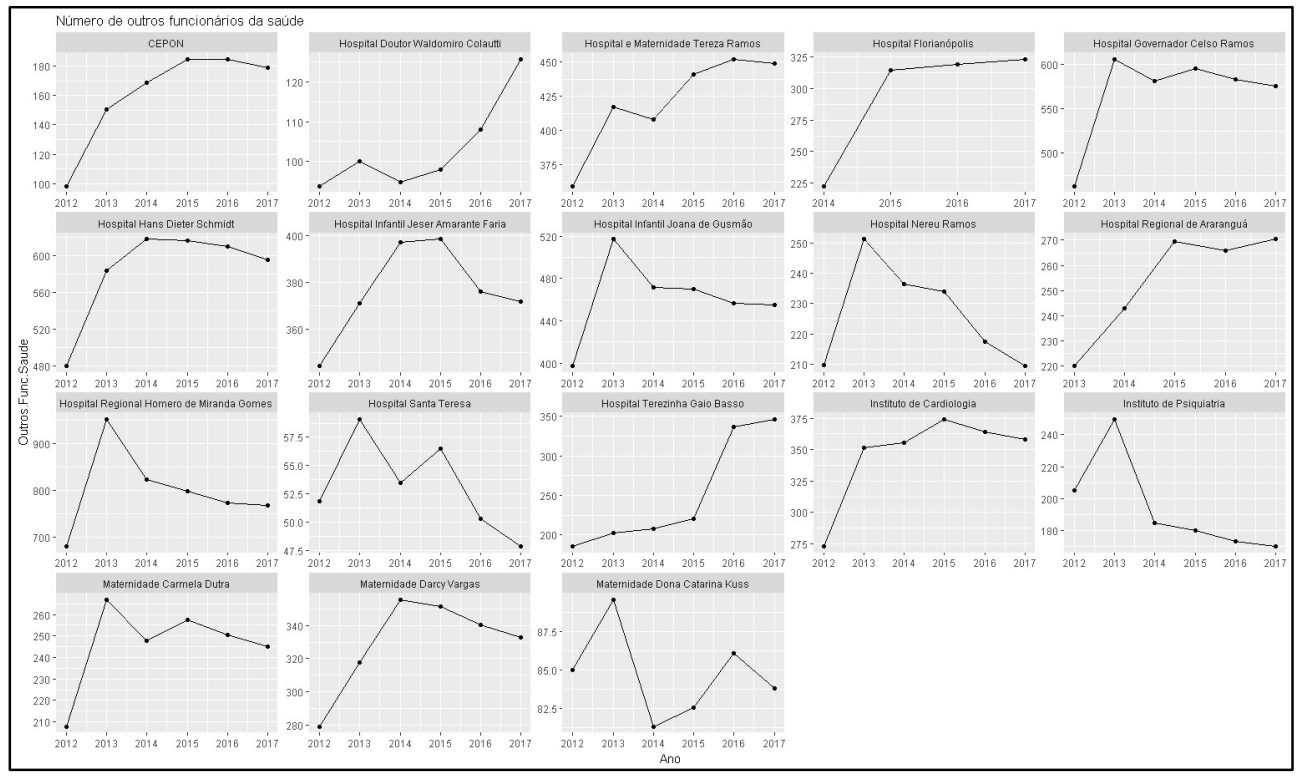

Figura 6: Evolução, por hospital, do número de outros funcionários entre 2012 e 2017. Fonte: Elaborada a partir de dados da SESSC e do CNES.

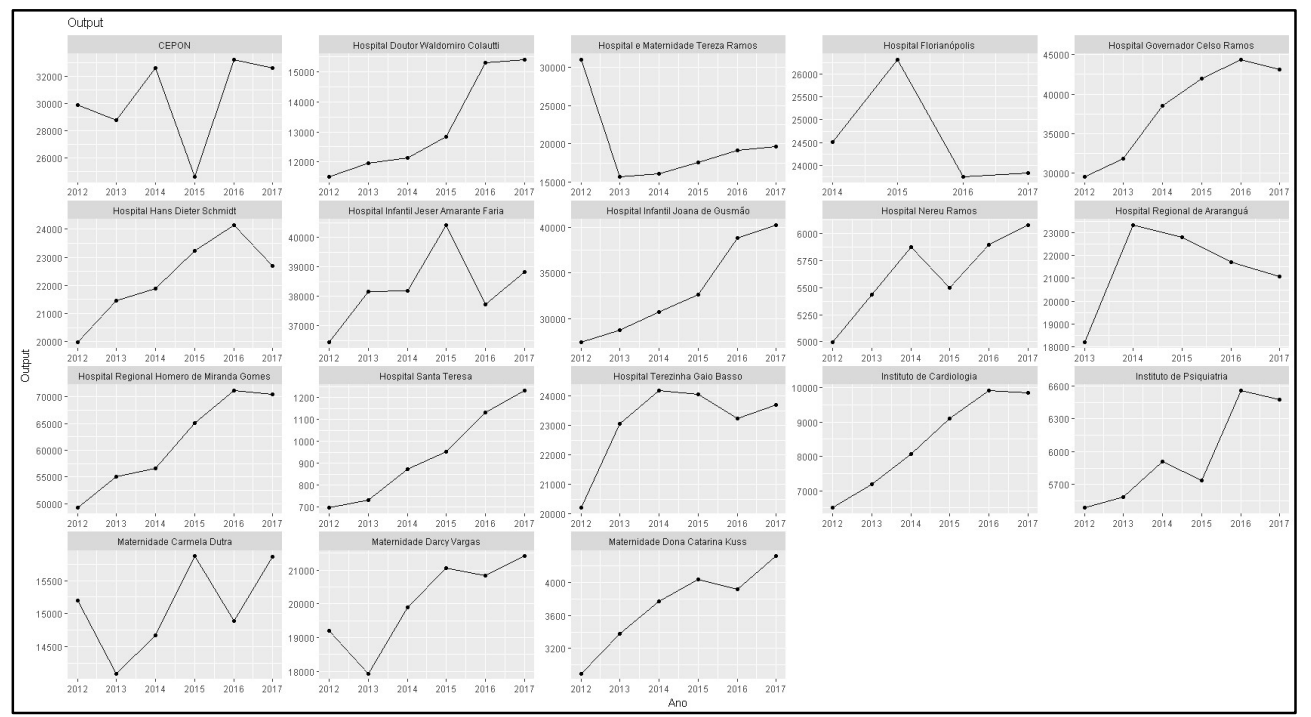

Figura 7: Evolução, por hospital, do número de serviços hospitalares entre 2012 e 2017. Fonte: Elaborada a partir de dados da SESSC e do CNES.

O CEPON aumentou em $80 \%$ o número de outros funcionários da saúde, enquanto Terezinha Gaio Basso aumentou 87\%. Aumentos significativos também ocorreram no Hospital Doutor Waldomiro Coulatti, Maternidade Tereza Ramos, Hospital Florianópolis, Hospital Celso Ramos, Hospital Hans Dieter Schmidt, Hospital Regional de Araranguá e Instituto de Cardiologia. O Hospital Nereu Ramos e a Maternidade Catarina Kuss mantiveram relativamente estável o número de outros funcionários da saúde, enquanto o Hospital Santa Tereza e o Instituto de Psiquiatria apresentaram pequenas quedas neste número. A Figura 7 apresenta 
a evolução, por hospital, do número ponderado de serviços hospitalares (outputs) utilizados no método DEA deste estudo, entre 2012 e 2017.

A figura acima indica que, embora não seja unânime, os hospitais vêm aumentando sua produção. 0 Hospital Doutor Waldomiro Colautti, o Hospital Celso Ramos, Hans Dieter Schmidt, Joana de Gusmão, Nereu Ramos, Hospital Regional Homero de Miranda Gomes, Hospital Santa Tereza, Hospital Teresinha Gaio Basso, Instituto de Cardiologia, Instituto de Psiquiatria, Maternidade Darcy Vargas e Maternidade Dona Catarina Kuss produziram mais, enquanto o Hospital e Maternidade Tereza Ramos e o Hospital Florianópolis encerraram o primeiro semestre de 2017 produzindo menos que sua produção de 2012. Embora dados a respeito do tempo médio de permanência não estejam incluídos na análise do modelo DEA, informações a respeito desta variável são importantes. A Figura 15 apresenta a evolução, por hospital, do tempo médio de permanência entre 2012 e 2017.

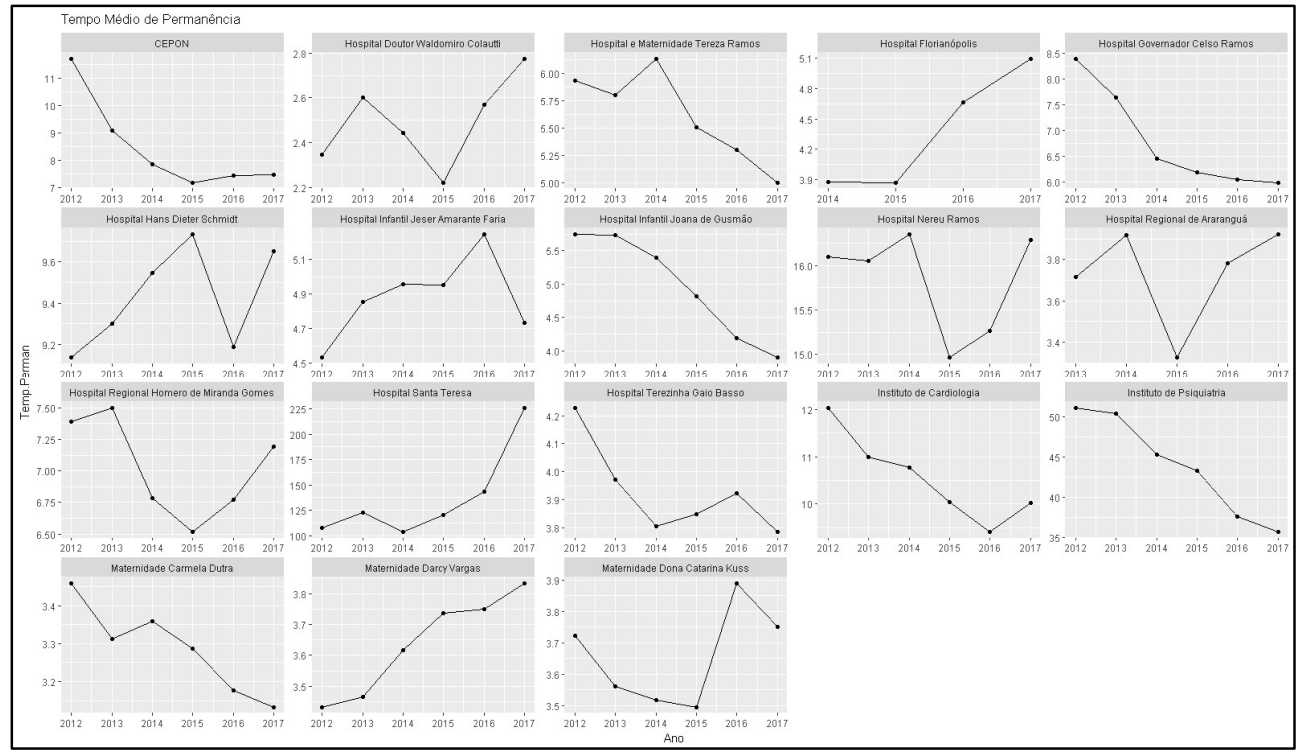

Figura 8: Evolução do tempo médio de permanência dos pacientes nas unidades hospitalares de 2012 a 2017. Fonte: Elaborada a partir de dados da SESSC e do CNES.

Para tanto, as variáveis selecionadas que serviram como inputs no modelo de Análise Envoltória de Dados foram: Quantidade de leitos habilitados; Número de médicos; Gastos por leito; úmero de outros funcionários da saúde (o que inclui enfermeiros, técnicos de enfermagem, auxiliares de enfermagem, nutricionistas, farmacêuticos, bioquímicos, fonoaudiólogos, etc.). Para o output, também foi considerado a variável de produção apresentada no capítulo anterior, que foi calculado a partir de ponderações entre os diferentes serviços da produção hospitalar (atendimentos emergenciais, ambulatoriais, internações, cirurgias e exames) baseado em sua relevância e custo para a saúde pública, de acordo com os contratos de gestão de hospitais elaborado pela Secretaria de Saúde do Estado. A matriz de correlações lineares de Pearson entre os inputs e o output são apresentadas na Figura 9. As correlações positivas estão em azul e as negativas em vermelho. 


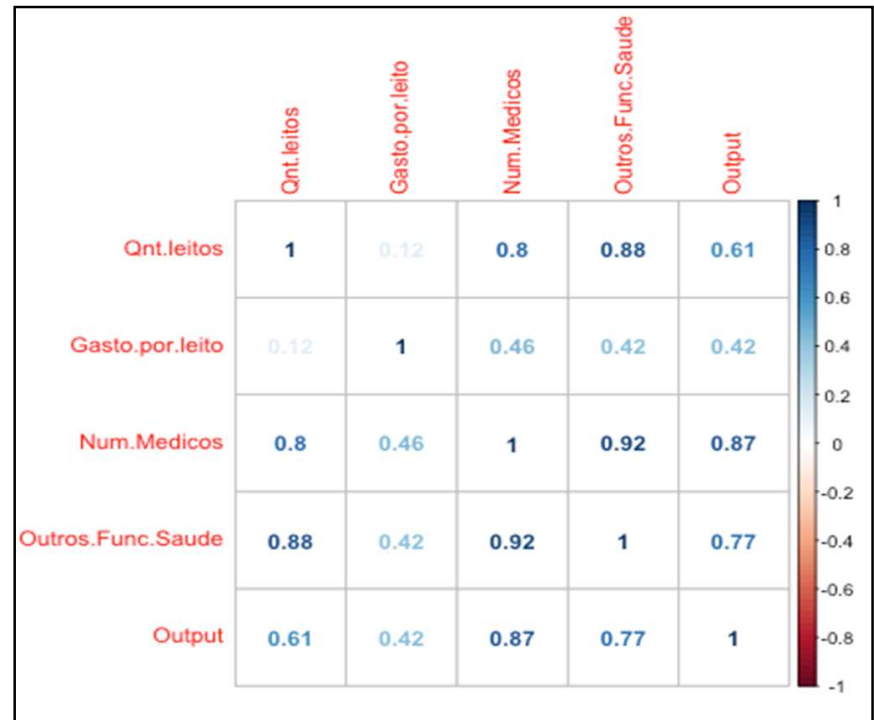

Figura 9: Matriz de correlação linear entre as variáveis. Fonte: Elaborada a partir de dados da SESSC e do CNES.

A figura acima evidencia que todas as correlações entre as variáveis são positivas. Entretanto, os valores de correlação que mais interessa para o DEA são dos inputs com a variável de output. Estas são equivalente a $0.61,0.42,0.87$, e 0.77 , isto é, todas as correlações são de moderadas a alta. A tabela 1 expõe os escores de eficiência dos hospitais estaduais de 2012 a 2017. É interessante se observar tanto comparativamente aos demais hospitais quanto a evolução do escore de eficiência ao longo do período. Ressalva-se que, para 2017, foram considerados dados somente de janeiro a junho.

Tabela 1: Escores de Eficiência para os 18 hospitais (2012 a 2017).

\begin{tabular}{|l|l|l|l|l|l|l|l|}
\hline Hospitais & $\mathbf{2 0 1 2}$ & $\mathbf{2 0 1 3}$ & $\mathbf{2 0 1 4}$ & $\mathbf{2 0 1 5}$ & $\mathbf{2 0 1 6}$ & $\mathbf{2 0 1 7}$ & Média \\
\hline Hospital Gov. Celso Ramos & 0,539 & 0,550 & 0,622 & 0,700 & 0,808 & 0,781 & 0.66667 \\
\hline Hospital Nereu Ramos & 0,183 & 0,225 & 0,264 & 0,285 & 0,258 & 0,265 & 0.24667 \\
\hline Hospital Regional Homero Gomes & 1,000 & 0,900 & 0,871 & 0,977 & 1,000 & 1,000 & 0.95800 \\
\hline Instituto de Cardiologia & 0,171 & 0,205 & 0,220 & 0,284 & 0,261 & 0,250 & 0.23183 \\
\hline Maternidade Carmela Dutra & 0,478 & 0,444 & 0,436 & 0,536 & 0,477 & 0,505 & 0.47933 \\
\hline Maternidade Darcy Vargas & 0,608 & 0,524 & 0,560 & 0,673 & 0,658 & 0,662 & 0.61417 \\
\hline Maternidade Dona Catarina Kuss & 0,164 & 0,255 & 0,240 & 0,367 & 0,253 & 0,283 & 0.26033 \\
\hline Hospital e Maternidade Tereza Ramos & 0,870 & 0,364 & 0,378 & 0,450 & 0,517 & 0,540 & 0.51983 \\
\hline Hospital Doutor Waldomiro Colautti & 0,700 & 0,805 & 0,751 & 1,000 & 0,875 & 0,813 & 0.82400 \\
\hline Instituto de Psiquiatria & 0,267 & 0,351 & 0,464 & 0,536 & 0,462 & 0,491 & 0.42850 \\
\hline Hospital Hans Dieter Schmidt & 0,474 & 0,425 & 0,431 & 0,450 & 0,507 & 0,483 & 0.46167 \\
\hline Hospital Santa Teresa & 0,094 & 0,172 & 0,185 & 0,268 & 0,221 & 0,241 & 0.19683 \\
\hline Hospital Infantil Joana de Gusmão & 0,574 & 0,647 & 0,623 & 0,691 & 0,834 & 0,833 & 0.70033 \\
\hline CEPON & 1,000 & 1,000 & 1,000 & 1,000 & 1,000 & 1,000 & 1.0000 \\
\hline Hospital Infantil Jeser Amarante Faria & 0,950 & 0,916 & 0,858 & 0,927 & 1,000 & 1,000 & 0.94183 \\
\hline Hospital Terezinha Gaio Basso & 1,000 & 1,000 & 1,000 & 1,000 & 1,000 & 1,000 & 1.0000 \\
\hline Hospital Regional de Araranguá & - & 0,789 & 0,821 & 0,909 & 0,981 & 0,894 & 0.87880 \\
\hline Hospital Florianópolis & - & - & 0,910 & 1,000 & 0,932 & 0,909 & 0.93775 \\
\hline
\end{tabular}

Fonte: Elaborada a partir de dados da SESSC e do CNES.

A Figura 10 apresenta os valores da tabela 1 de forma gráfica, expondo visualmente a evolução dos escores de eficiência para cada hospital. Para facilitar a visualização dos resultados, são apresentadas a seguir as figuras 11 e 12, as quais demonstram a evolução ao longo do tempo dos índices de eficiência mensurados por meio do DEA. As figuras a seguir apresentam os resultados para os hospitais de gestão própria e para os administrados os OSS, respectivamente. 


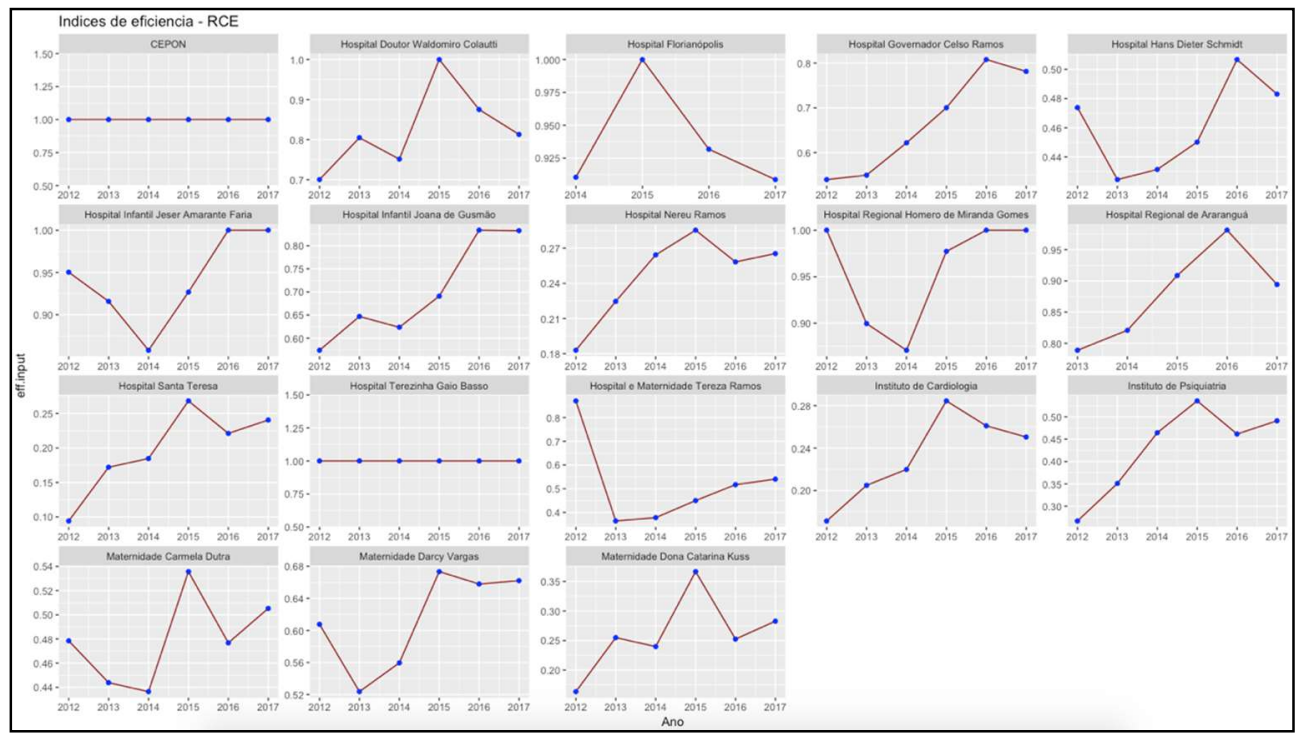

Figura 10: Evolução dos Escores de Eficiência dos hospitais (2012 a 2017). Fonte: Elaborada a partir de dados da SESSC e do CNES.

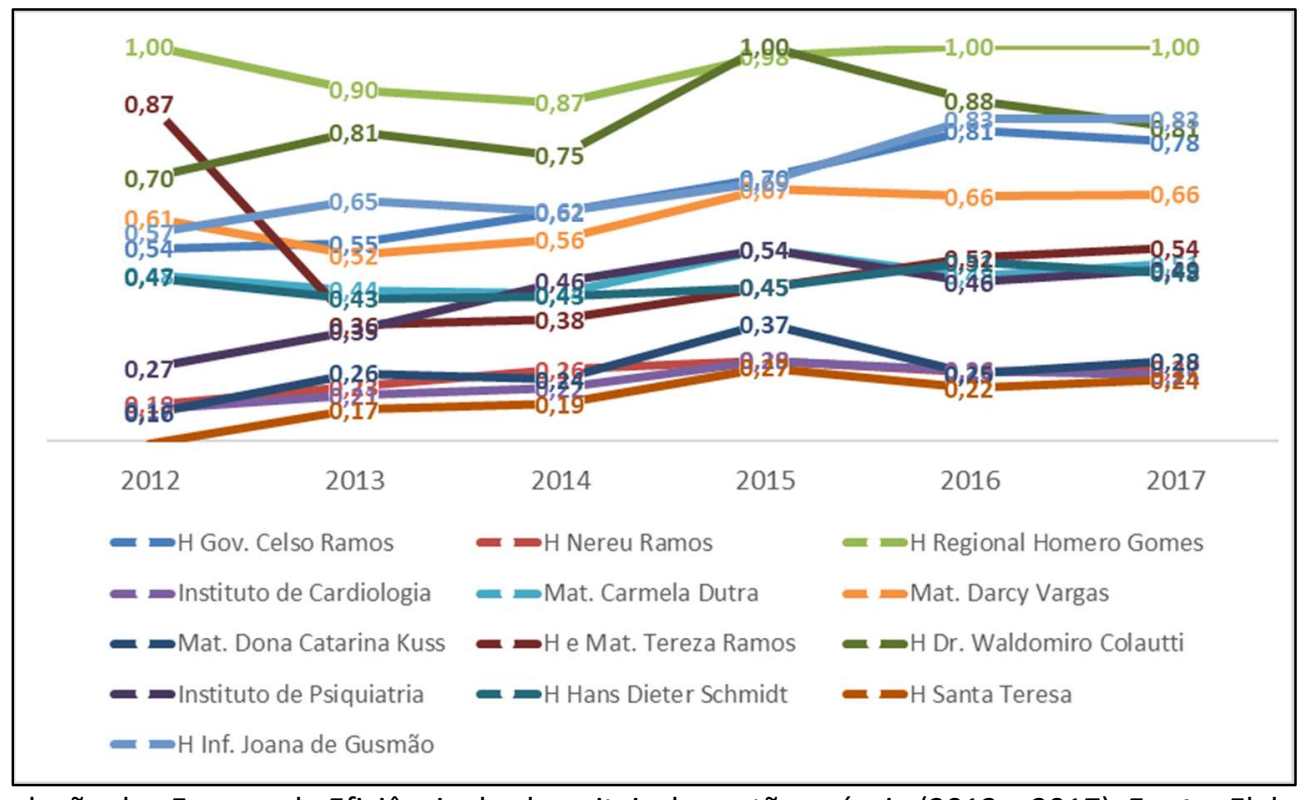

Figura 11: Evolução dos Escores de Eficiência dos hospitais de gestão própria (2012 a 2017). Fonte: Elaborada a partir de dados da SESSC e do CNES.

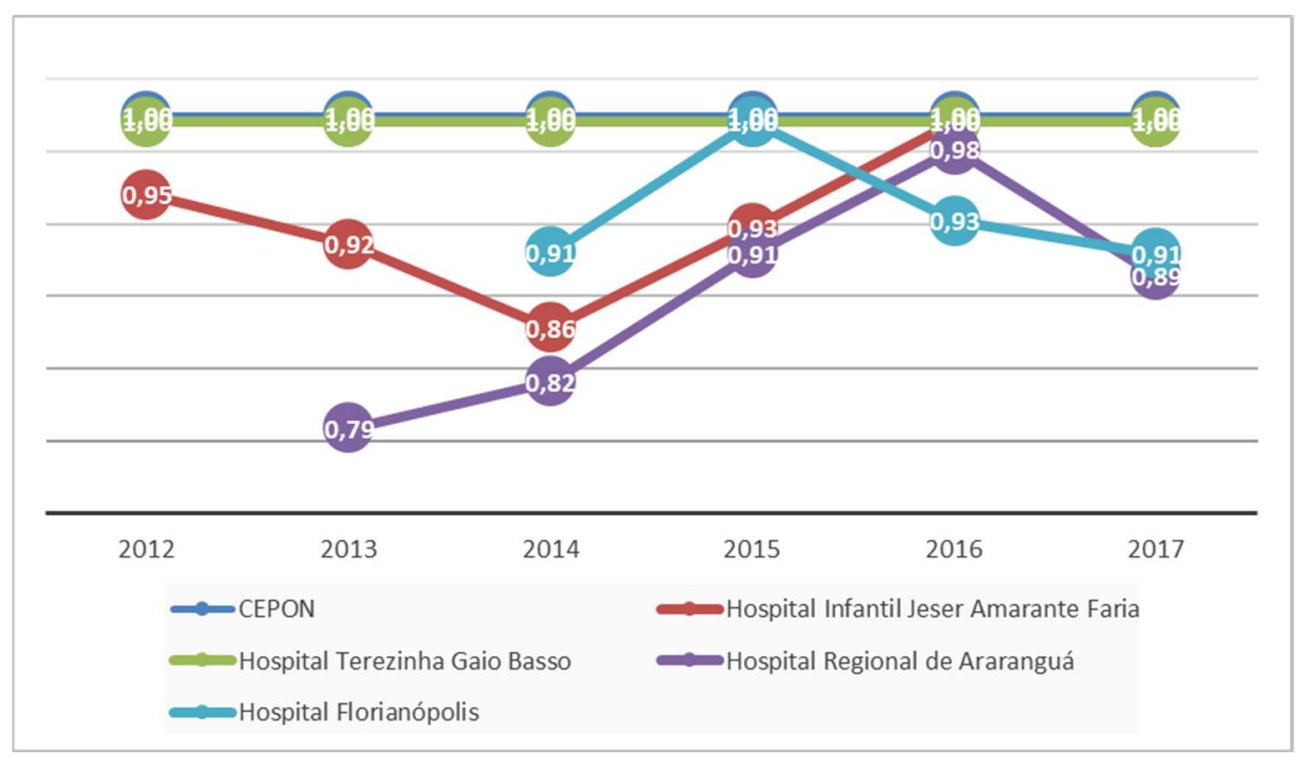

Figura 12: Evolução dos Escores de Eficiência dos hospitais geridos por OS (2012 a 2017). Fonte: Elaborada a partir de dados da SESSC e do CNES. 
Com base na Tabela 1 e nas figuras apresentadas anteriormente, destaca-se os seguintes pontos: CEPON e o Hospital Teresinha Gaio Basso são os hospitais públicos estaduais mais eficientes, e apresentaram valores máximos de eficiência relativa em todos os anos; O Hospital Infantil Jeser Amarante Faria, também gerido por OSS, melhorou sua gestão em termos de eficiência relativa em 2016 e em 2017. Já 2014 foi o ano mais ineficiente deste hospital, mas sua média de eficiência para o período foi alta, equivalendo a 0,942; Os hospitais geridos por OSS menos eficientes são o Hospital Florianópolis e o Hospital Regional de Araranguá (embora superiores em termos de eficiência a grande parte dos hospitais da administração direta). Seus escores médios de eficiência foram 0,937 e 0,878, respectivamente. Nota-se que o Hospital Florianópolis teve em 2015 seu ano mais eficiente, mas depois apresentou queda nos índices de eficiência. Já o Hospital Regional de Araranguá apresentou o melhor resultado em 2016. Sua performance de eficiência foi crescente até 2016 e decrescente em 2017. Ambos hospitais são geridos pela mesma organização social, a SPDM, cuja sede administrativa fica em São Paulo. Em 2017, estes foram os únicos hospitais geridos por OSS que não atingiram a eficiência máxima; O Hospital Celso Ramos apresentou média de escores de eficiência de 0,666. Este hospital, com exceção de 2017, vem se tornando mais eficiente ao longo dos anos; A Maternidade Darcy Vargas é o quinto hospital público estadual mais eficiente gerido pela administração direta, e é a maternidade com mais altos escores de eficiência. Sua média foi equivalente a 0,614. Nota-se que a Maternidade Darcy Vargas passou a ser significamente mais eficiente a partir de 2015, e se mantendo estável desde então; Com um escore médio de 0,519, o Hospital e Maternidade Tereza Ramos é o sexto hospital mais eficiente deste modelo organizacional de gestão. Em 2012 este hospital apresentou alto escore de eficiência relativa, 0,87. Mas em 2013 houve uma forte queda neste índice, caindo para 0,364. Desde então, os escores de eficiência vem melhorando, mas 2017 ainda estava longe do seu patamar de 2012, estando equivalente a 0,54; 0 próximo hospital a citar é a Maternidade Carmela Dutra, com escore médio de 0,479. Esta maternidade reduziu sua eficiência de 2012 a 2014, e em 2015 apresentou seu melhor índice; A oitava posição nesta categoria de gestão fica com o Hospital Hans Dieter Schmidt e seus 0,462 pontos médios de eficiência. Em 2013, este hospital foi menos eficiente que em 2012, mas, desde então, vem melhorando seus índices de eficiência, sendo que em 2017 houve uma leve redução no escore em relação ao ano anterior; O Instituto de Psiquiatria (IPQ) e seus 0,4285 pontos médios de eficiência os colocam em nono lugar de hospitais públicos estaduais geridos pela administração direta mais eficientes. Embora o IPQ seja um hospital com baixo índice de eficiência média, nota-se que, de 2012 a 2015, houve expressiva melhora na eficiência relativa do hospital; O Hospital Nereu Ramos é o antepenúltimo hospital público estadual menos eficiente. Embora seu escore de eficiência tenha apresentado tendência crescente de 2012 a 2015, este mesmo caiu em 2016 e relativamente estabilizou na primeira metade de 2017. Seu escore médio foi de 0,247. A Figura 12 apresenta um comparativo anual de escores médios de eficiência relativa entre os dois modelos de gestão organizacional. 


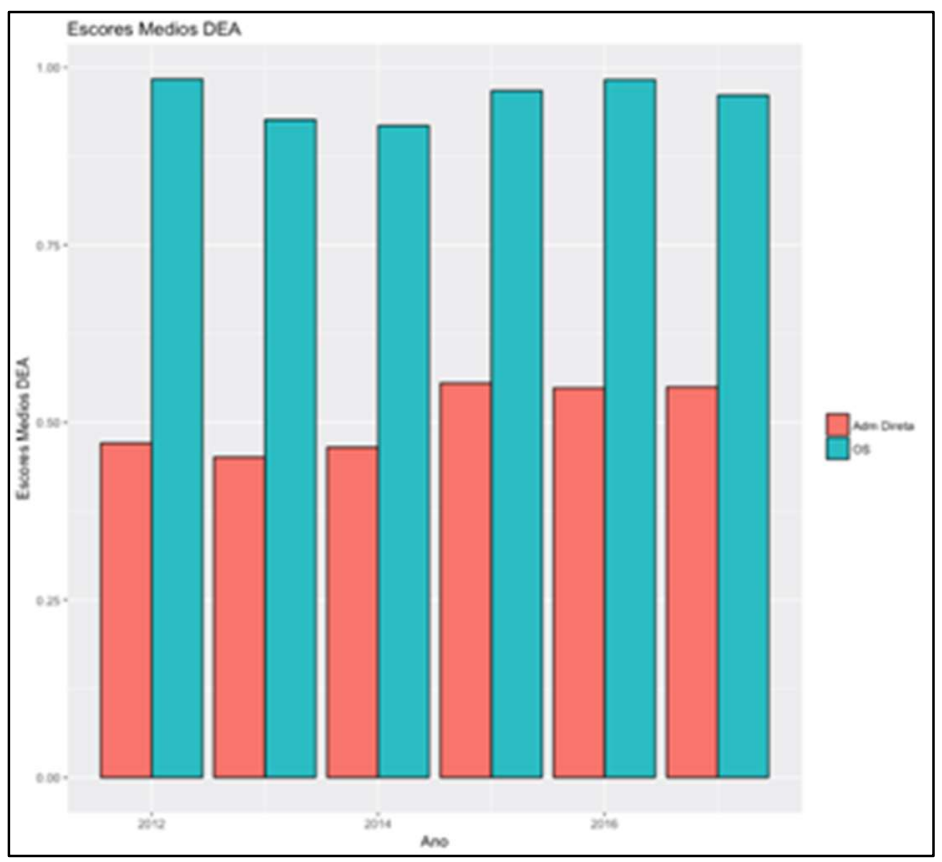

Figura 12: Comparativo de Escores de Eficiência entre Modelos de Gestão. Fonte: Elaborada a partir de dados da SESSC e do CNES.

A figura acima mostra em verde os escores médios dos hospitais gerenciados por OSS, e em laranja os escores médios de eficiência relativa dos hospitais da administração direta. Evidencia-se assim, a hipótese levantada no início do presente estudo: em termos de eficiência, os hospitais geridos por Organizações Sociais de Saúde apresentam escores significativamente superior aos hospitais geridos pela administração direta. Isto ocorre em todos os anos analisados no estudo. A Figura 12 permite também observar que o ano de 2013 e 2014 foram os anos menos eficientes na gestão global dos hospitais estaduais.

Uma vantagem da Análise Envoltória de Dados é emitir informações quanto ao benchmarking de hospitais eficientes para hospitais ineficientes. Assim sendo, aplicou-se uma análise de benchmarking nos anos de 2016 e 2017. Contatou-se uma consistência nos resultados de benchmarking, sendo que das 56 possibilidades de cálculo em comum entre 2016 e 2017, 54 delas emitiram os mesmos resultados nos dois anos (o que equivale a 96,5\% de semelhança de resultados entre 2016 e 2017). A tabela 2 enumera os hospitais estaduais públicos de 1 a 18, e relaciona os hospitais-referência (peers) para os anos de 2016 e 2017. O hospital dado como 'Peer 1' tem um peso de referência superior ao hospital dado como 'Peer 2'. Hospitais que atingiram escore de eficiência igual a 1 no período não possui peers.

Tabela 2: Análise de hospitais de referência.

\begin{tabular}{|c|c|c|c|c|c|}
\hline \multicolumn{2}{|c|}{ Hospitais } & \multicolumn{2}{|l|}{2016} & \multicolumn{2}{|l|}{2017} \\
\hline & & Peer 1 & Peer 2 & Peer 1 & Peer 2 \\
\hline 1 & Hospital Celso Ramos & 5 & 15 & 15 & 16 \\
\hline 2 & Maternidade Carmela Dutra & 15 & 16 & 15 & 16 \\
\hline 3 & Hospital Nereu Ramos & 15 & 17 & 15 & 17 \\
\hline 4 & Hospital Infantil Joana de Gusmão & 15 & 16 & 15 & 16 \\
\hline 5 & Hospital Regional Homero de Miranda Gomes & - & - & - & - \\
\hline 6 & Instituto de Cardiologia & 15 & 17 & 15 & 17 \\
\hline 7 & Instituto de Psiquiatria & 15 & - & 15 & - \\
\hline 8 & Hospital Hans Dieter Schmidt & 5 & 17 & 5 & 17 \\
\hline 9 & Maternidade Darcy Vargas & 5 & 15 & 5 & 15 \\
\hline 10 & Hospital Santa Teresa & - & - & - & - \\
\hline 11 & Hospital e Maternidade Tereza Ramos & 5 & 15 & 5 & 15 \\
\hline
\end{tabular}




\begin{tabular}{|l|l|l|l|l|l|}
\hline 12 & Hospital Doutor Waldomiro Coulatti & 15 & - & 15 & 16 \\
\hline 13 & Maternidade Dona Catarina Kuss & 15 & - & 15 & - \\
\hline 14 & Hospital Florianópolis & 15 & 16 & 15 & 16 \\
\hline 15 & CEPON & - & - & - & - \\
\hline 16 & Hospital Infantil Jeser Amarante Faria & - & - & - & - \\
\hline 17 & Hospital Terezinha Gaio Basso & - & - & - & - \\
\hline 18 & Hospital Regional de Araranguá & 5 & 15 & 5 & 15 \\
\hline
\end{tabular}

A tabela 2 constata que existem 4 hospitais-referência dentro do estado de Santa Catarina. Extrai-se também as seguintes conclusões: CEPON é hospital-referência o maior número de vezes, aparecendo 24 vezes no quadro 12, sendo que destas, 17 vezes como peer 1; O Hospital Regional Homero de Miranda Gomes foi referência 9 vezes entre 2016 e 2017, sendo o segundo hospital com maior número de referências no quadro 12. Foi inclusive o Peer 1 dos hospitais Celso Ramos, Hans Dieter Schmidt, Hospital e Maternidade Tereza Ramos e Hospital Regional de Araranguá (uma OSS); O Hospital Infantil Jeser Amarante Faria foi o terceiro hospital mais citado como referência, com 8 vezes sendo tipo como hospital-referência, inclusive como referência para o Hospital Infantil Joana de Gusmão em ambos 2016 e 2017; O último hospitalreferência foi o Hospital Terezinha Gaio Basso, com 6 referências; O único hospital ineficiente que não resultou em nenhum peer foi o Hospital Santa Teresa. Isto provavelmente deve-se aos baixíssimos escores de eficiência apresentados no período, que pode ter impossibilitado o cálculo de benchmarking do DEA.

Pelos resultados de benchmarking, pode constatar que, embora as OSS apresentem resultados de eficiência média superiores aos outros hospitais, a gestão também é um fator muito forte para os resultados, sendo evidenciado pelo benchmarking apresentado do Hospital Regional Homero de Miranda Gomes.

\section{CONCLUSÕES}

Segundo La Forgia et al. (2008), é recomendado que se desenvolva uma estratégia, um marco regulatório e um plano de implementação para converter os estabelecimentos da administração direta e indireta em arranjos organizacionais alternativos que propiciem autonomia decisória e gerenciamento flexível dos recursos humanos, como as OSS. Em Santa Catarina, são 5 hospitais geridos por OSS e pretendese estender este modelo organizacional para outros hospitais. Constatou-se que as OSS, em Santa Catarina, são, em média, $46 \%$ mais eficientes que os hospitais geridos pela administração direta. Os autores também recomendam uma política de investimentos que promova a adoção de arranjos organizacionais autônomos em todo hospital público novo.

Embora os hospitais geridos por OSS tenham se mostrado superiores no que tange à eficiência do gasto público, observou-se que aspectos gerenciais também importam muito na qualidade do gasto, como foi evidenciado por alguns hospitais da administração direta com altos escores de eficiência. $O$ que é mais relevante é um forte, contínua e autêntica fiscalização dos hospitais com clara definição de funções e responsabilidades, e de modo que as ações necessárias para cumprir obrigações sejam claramente compreendidas. A confiabilidade e auditoria dos dados emitidos pelos hospitais, e um claro alinhamento das expectativas de desempenho, tornam mais fácil de identificar e corrigir deficiências.

Sobre a possibilidade de transferir a gestão dos atuais hospitais de administração estadual para as 
OSS, sabe-se que a implementação de reformas organizacionais no setor público é uma tarefa altamente complexa. As OSS de São Paulo, por exemplo, foram introduzidas apenas em unidades novas devido à resistência dos hospitais já estabelecidos em mudar o seu modelo de gestão. Em uma análise da experiência europeia, Maarse et al. (2005; citado por LA FORGIA et al., 2009). Fazem um resumo sobre o processo de reforma dos hospitais:

Em muitos países, vê-se forte resistência à realização de reformas em larga escala. O padrão comum parece ser os governos procederem em passos gradativos. Hospitais com estruturas orçamentárias foram convertidos em hospitais autônomos (ex. controle sobre os recursos materiais), que por sua vez têm sido transformados em modelos totalmente (controle sobre todos os insumos, inclusive dos recursos humanos). Muitos governos, parece, continuam convencidos de que os hospitais devem preservar sua condição de instituições públicas e que a privatização irá minar os objetivos públicos de controle de custo, equidade e acessibilidade.

Constata-se a necessidade de um estudo sobre formas de remuneração por desempenho que levem em conta o potencial de produção dos hospitais, ao contrário de metas por médias históricas de produção. Um estudo econométrico específico sobre as metas, juntamente com auditorias minuciosas nos hospitais poderiam fazer parte da estipulação de novas metas que contemplem insumos em capacidades ociosas. São necessários fortes incentivos à melhoria da qualidade e da eficiência.

\section{REFERÊNCIAS}

COELLI, T.; PRASADA, R. D.; O'DONNEL, C.; BATTESE, G.. An introduction to efficiency and productivity analysis. New York: Springer, 2005.

EMROUZNEJAD, A.; YANG, G.. A survey and analysis of the first 40 years of scholarly literature in DEA: 1978-2016. Socio-Economic Planning Sciences, v.61, p.4-8, 2018. DOI: https://doi.org/10.1016/j.seps.2017.01.008

GIOVANELLA, L.; ESCOREL, S.; LOBATO, L.; NORONHA, J.; CARVALHO, A.. Políticas e sistema de saúde no Brasil. Rio de Janeiro: Fiocruz 2012.

GONÇALVES, A. C.; NORONHA, C. P.; LINS, M. P. E.; ALMEIDA, R. M. V. R.. Análise Envoltória de Dados na avaliação de hospitais públicos nas capitais brasileiras. Revista Saúde Pública, v.41, n.3, p.427-35, 2007.

GRIGOLI, F.; KAPSOLI, J.. Waste not, want not: the efficiency of health expenditure in emerging and developing economies. IMF Working Paper. Washington: Fundo Monetário Internacional, 2013.

KIRIGIA, J. M.; EMROUZNEJAD, A.; SAMBO, L. G.; MUNGUTI, N.; LIAMBILA, W.. Using data envelopment analysis to measure the technical efficiency of public health centers in Kenya. Journal of Medical Systems, v.28, p.155-166, 2004.

LA FORGIA; G. M.; COUTTOLENC, B.. Hospital Performance in Brazil: The Search for Excellence. Washington: Banco Mundial, 2008.

MARINHO, E.; BARRETO, F.; LIMA, F.. Produtividade, variação tecnológica e variação de eficiência técnica das regiões e estados brasileiros. In: ENCONTRO NACIONAL DE ECONOMIA, 29. Anais. Rio de Janeiro: ANPEC, 2001.

MELLO, J.; MEZA, L.; GOMES, E.; NETO, L.. Curso de análise envoltória de dados. In: SIMPÓSIO BRASILEIRO DE PESQUISA OPERACIONAL, 37. Anais. 2005.

SMITH, P. C.; STREET, A.. Measuring the efficiency of public services: the limits of analysis. Journal of the Royal Statistical Society, v.168, n.2, p.401-417, 2005.

THANASSOULIS, E.. Introduction to the theory and application of data envelopment analysis: a foundation text with integrated software. New York: Springer Science, 2001.

A CBPC - Companhia Brasileira de Produção Científica (CNPJ: 11.221.422/0001-03) detém os direitos materiais desta publicação. Os direitos referem-se à publicação do trabalho em qualquer parte do mundo, incluindo os direitos às renovações, expansões e disseminações da contribuição, bem como outros direitos subsidiários. Todos os trabalhos publicados eletronicamente poderão posteriormente ser publicados em coletâneas impressas sob coordenação da Sustenere Publishing, da Companhia Brasileira de Produção Científica e seus parceiros autorizados. Os (as) autores (as) preservam os direitos autorais, mas não têm permissão para a publicação da contribuição em outro meio, impresso ou digital, em português ou em tradução. 